\title{
Soluble glucocorticoid-induced TNF receptor (sGITR) induces inflammation in mice
}

\author{
Hyun-Hee Shin ${ }^{1}$, Suk-Gi Kim ${ }^{1}$ \\ Moo-Hyung Lee ${ }^{1}$, Jae-Hee Suh ${ }^{2}$ \\ Byoung S. Kwon ${ }^{1}$ and Hye-Seon $\mathrm{Choi}^{1,3}$ \\ ${ }^{1}$ Department of Biological Sciences \\ and the Immunomodulation Research Center \\ University of Ulsan, Ulsan 680-749, Korea \\ ${ }^{2}$ Department of Diagnostic Pathology \\ Ulsan University Hospital, Ulsan, Korea \\ ${ }^{3}$ Corresponding Author: Tel, 82-52-259-1545; \\ Fax, 82-52-259-1694; E-mail, hschoi@mail.ulsan.ac.kr
}

Accepted 31 July 2003

Abbreviations: GITR, glucocorticoid-induced tumor necrosis factor receptor; HRP, horseradish peroxidase; TNFR/NGFR, TNF/nerve growth factor receptor

\begin{abstract}
Glucocorticoid-induced TNF receptor (GITR) was a new member of the TNF/nerve growth factor receptor (TNFR/ NGFR) family and induced in murine $T$ cells by dexamathasone. Recombinant soluble GITR (sGITR) induced an inflammation in peritoneal membrane and changes in spleen after i.p. injection of $3 \mathrm{mg} / \mathrm{kg}$ in C57BL/6 mice. Spleen was enlarged and percentage of neutrophils and monocytes were increased. The area of red pulp in spleen was increased, while that of white pulp was decreased after GITR injection. The thickening of membrane and neutrophil infiltration was observed in peritoneal membrane with increased myeloperoxidase activity. At later time, neutrophil infiltration moved to inside the tissue with tissue damage. GITR ligand and GITR were expressed constitutively on the surface of spleen cells and cells from peritoneal fluid. In contrast, no significant change in the spleen and in peritoneal membrane was observed in mice treated with LPS. GITR may play a role in body's inflammatory processes.
\end{abstract}

Keywords: dexamathasone; glucocorticoids; inflammation; receptors, tumor necrosis factor

\section{Introduction}

Glucocorticoid-induced TNF receptor (GITR) was described as a new member of the TNF/nerve growth factor receptor (TNFR/NGFR) family and induced in murine $\mathrm{T}$ cell hybridoma 3D0 cells by dexamathasone (Nocentini et al., 1997). TR11 (AITR) isolated from a human activated T cell cDNA library has 55\% identity with murine GITR at the amino acid level (Kwon, et al., 1999). The high sequence conservation between human and mouse suggests that TR11 is the human homologue of GITR. They may, however, have different biological activities because of following reasons; there is a mismatch in the first cysteine rich pseudorepeat between GITR and TR11, and TR11 is not inducible by dexamethasone. GITR shows a similarity in amino acid sequence of intracellular tails to those of 4-1BB, CD27, and TR11 (Kwon and Weismann, 1989; Jacquot et al., 1997; Heinisch et al., 2000; Takeda et al., 2000). It has been reported that TR11, 4-1BB, and CD27 transmit their signals through TRAF2 to NF- $\mathrm{KB}$ (Akiba et al., 1998; Arch and Thompson, 1998; Jang et al., 1998; Kwon, et al., 1999; Cannons et al., 2000).

Glucocorticoid hormones control the host immune responses to infection and tissue invasion and are generated in response to the systemic stress mediated by the hypothalamus-pituitary-adrenal axis. Glucocorticoids are known to be powerful anti-inflammatory agents which inhibit immune cell activation, proliferation, and cytokine productions (Goldstein et al., 1992).

In this study, we have shown that soluble GITR induces an inflammation in peritoneal membrane and changes in spleen after i.p. injection in C57BL/6 mice. GITR may play a role in body's inflammatory processes.

\section{Material and Methods}

\section{Mateials and reagents}

DMEM, FBS, penicillin, and streptomycin, LPS (from Escherichia coli serotype 055:B5), guanidine thiocyanate, and isopropyl $\beta$-D-1-thiogalactopyranoside (IPTG) were obtained from Sigma Chemical Co. (St. Louis, MO). mAb for histidine and E. coli BL21 strain were from Novagen (Madison, WI). FITC-conjugated goat anti-mouse $\lg$, FITC-conjugated goat anti-rabbit $\mathrm{IgG}$, and FITC-conjugated goat $\lg G$ were from 
Southern Biotech. (Birmingham, AL). A mouse recombinant protein, GITR-Fc, was from Alexis Corporation (UK). Recombinant extracellular domain of GITR (sGITR) was prepared in Escherichia coli-pET $28 \mathrm{a}$ and purified as described previously (Shin et al., 2002). The sGITR was purified by eluting the protein from $10 \%$ SDS-PAGE to avoid possible endotoxin contamination. SDS gel-purified rGITR containd no more than trace amount of endotoxin $(<0.4 \mathrm{EU} / \mathrm{mg}$ by E-Toxate assay from Sigma Chemical). Polyclonal antiserum against GITR was obtained from rabbit as described before (Shin et al., 2002)

A

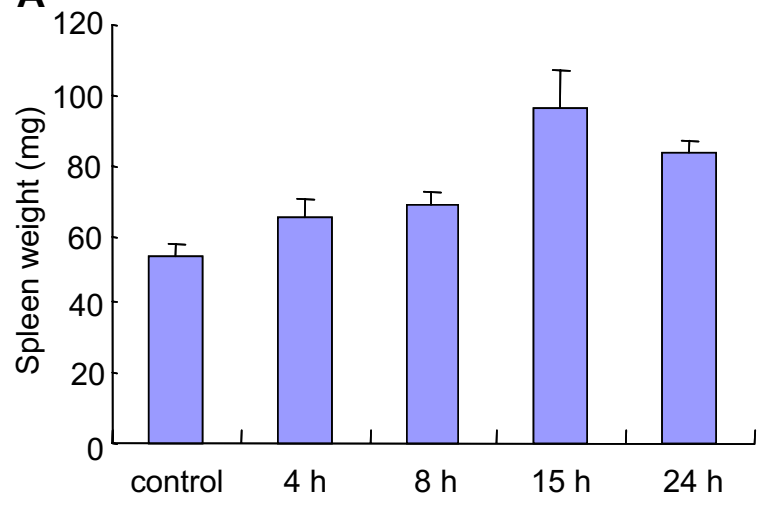

C

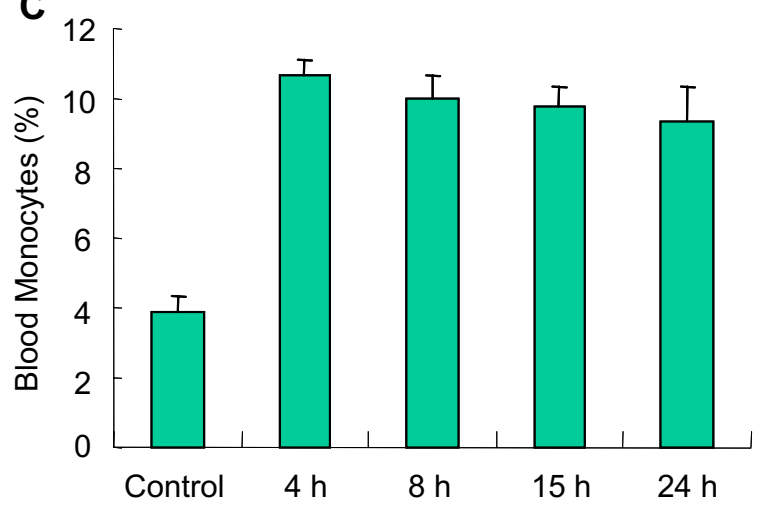

E

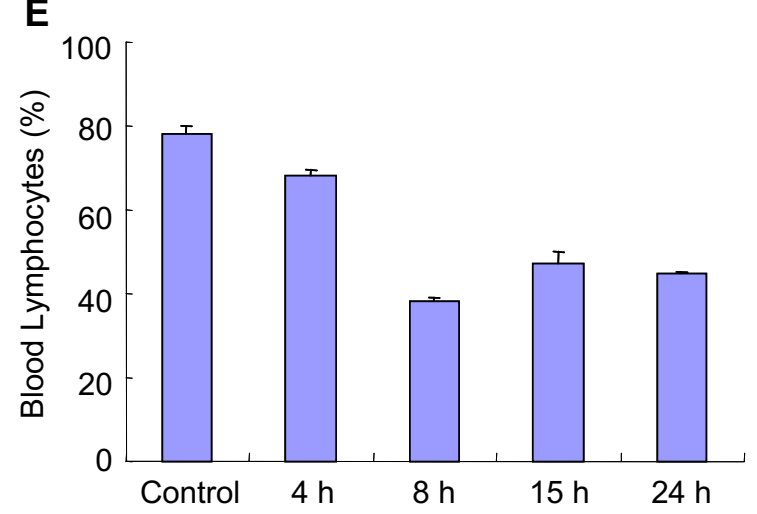

\section{Mice and treatment}

Seven-week-old male C57BL/6 mice $(-20 \mathrm{~g})$ were injected i.p. with $3 \mathrm{mg} / \mathrm{kg}$ of GITR or $50 \mathrm{mg} / \mathrm{kg}$ of LPS in saline. Control mice were injected with saline alone $(n=10)$. After $4 \mathrm{~h}(n=5), 8 \mathrm{~h}(n=6), 15 \mathrm{~h}(n$ $=7), 24 \mathrm{~h}(n=7)$ of GITR or $3 \mathrm{~h}(n=5), 15 \mathrm{~h}(n$ $=4$ ) of LPS, the mice were sacrificed, blood was drawn, and peritoneal membrane, spleen, and peritoneal lipids were obtained. Part of the tissues was stored in 10\% formalin, stained by hematoxylin and eosin, and photographed. Spleen was weighed and peritoneal membrane was homogenated, and cell

B

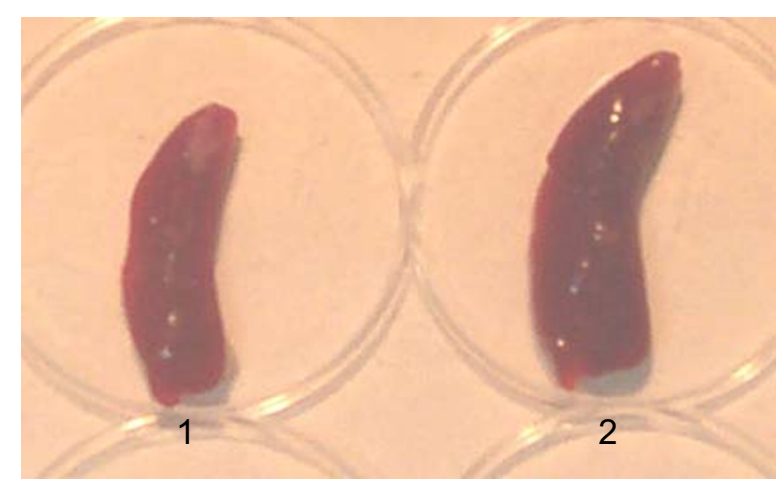

D

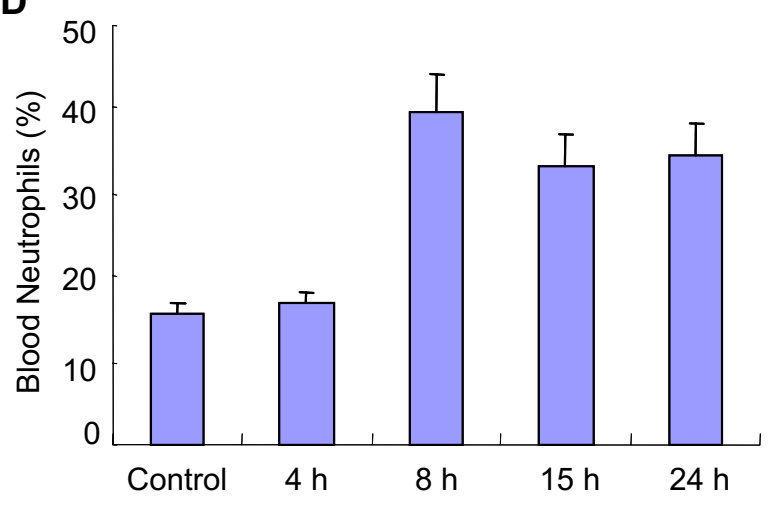

Figure 1. Profiles of spleen enlargements $(A, B)$, peripheral blood monocytes $(C)$, neutrophils $(C)$, and lymphocytes $(E)$ in $C 57 B L / 6$ Mice. Mice were administered i.p. with $3 \mathrm{mg} / \mathrm{kg}$ of rGITR. Control mice were injected with saline alone. After $4,8,15,24 \mathrm{~h}$ of rGITR, spleen was taken and weighed (B1; spleen from control, B2; spleen from $15 \mathrm{~h}$ of rGITR treated mice). Blood was drawn and analyzed by Hemavet. The \% monocytes, $\%$ neutrophils, and \% lymphocytes were of total leukocytes. Each sample was measured in triplicate. 
lysate was obtained for myeloperoxidase assay.

\section{Flow cytometry}

Cells $(5 \times 10 \%$ sample) were incubated in incubation buffer (PBS, $10 \%$ human serum and $0.1 \%$ sodium azide) with purifed polyhistidine- tagging SGITR or rabbit anti-GITR polyclonal $A b$ on ice for $30 \mathrm{~min}$ for detection of GITR ligand and GITR, respectively. Same volume of PBS and preimmune serum were used as a isotype control for GITR ligand and GITR, respectively. Cells were washed three times in PFS (phosphate buffered saline, $2.5 \%$ fetal bovine serum and $0.1 \%$ sodium azide). The cells were incubated on ice for $30 \mathrm{~min}$ with mouse anti-His $A b$ in incubation buffer for detection of GITR ligand. Cells were washed three times. Cells were then incubated on ice for $30 \mathrm{~min}$ with FITC-conjugated goat antimouse IgG or FITC-conjugated goat anti-rabbit IgG for detection of GITR ligand and GITR, respectively. The cells were washed again as above and flow cytometry was performed using a FACSCalibur (Becton Dickinson).

\section{Results}

\section{Effects of GITR in mice}

Previously extracellular domain of GITR activated macrophages to induce iNOS (Shin et al., 2002),
COX-2 (Shin et al., 2002), and MMP-9 (Lee et al., 2003). We tested the effects of soluble GITR (sGITR) kinetically in C57BL/6 mice. When mice were treated intraperitoneally with SGITR at a dose of $3 \mathrm{mg} / \mathrm{kg}$, spleen was enlarged (Figure 1A) and percentage of neutrophils and monocytes were increased in peripheral blood (Figure $1 \mathrm{C}$ and 1D) whereas control mice injected with PBS showed no significant changes. Spleen enlargement was eminent at $15 \mathrm{~h}$ after SGITR injection (Figure 1B). The percentage of neutrophil was maximum at $8 \mathrm{~h}$ and remained up to $24 \mathrm{~h}$ and that of monocyte reached maximum at $4 \mathrm{~h}$ and remained, while that of lymphocyte decreased (Figure 1E). Significant changes in spleen and peritoneal membrane was observed by histochemical analysis using hematoxylin and eosin staining. The area of red pulp in spleen was increased, while that of white pulp was decreased after sGITR injection (Figure 2A). The increase of red pulp in spleen was significant at $15 \mathrm{~h}$ and $24 \mathrm{~h}$ after sGITR injection. The thickening of membrane and neutrophil infiltration was observed in peritoneal membrane after $4 \mathrm{~h}$, and was maximum at $15 \mathrm{~h}$. The edema was decreased by $24 \mathrm{~h}$ after sGITR injection, but neutrophil infiltration moved to inside the tissue with tissue damage (Figure 3A). At the site of inflammation, neutrophils are among the first cells to arrive. Since activation of neutrophils results in myeloperoxidase into the extracellular matrix, myeloperoxidase level was determined in peritoneal membrane homoge-
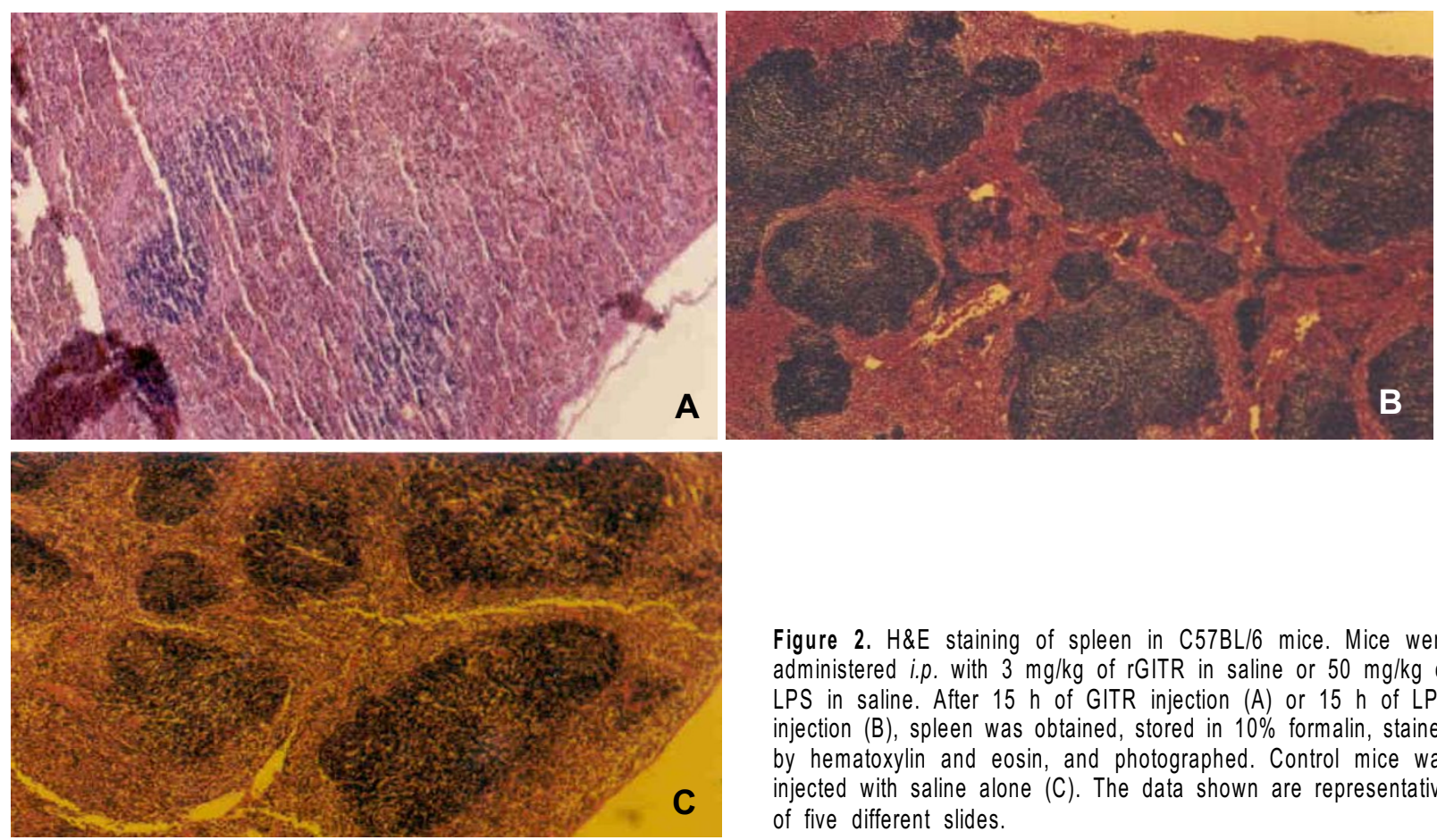

Figure 2. H\&E staining of spleen in C57BL/6 mice. Mice were administered i.p. with $3 \mathrm{mg} / \mathrm{kg}$ of rGITR in saline or $50 \mathrm{mg} / \mathrm{kg}$ of LPS in saline. After $15 \mathrm{~h}$ of GITR injection (A) or $15 \mathrm{~h}$ of LPS injection (B), spleen was obtained, stored in $10 \%$ formalin, stained by hematoxylin and eosin, and photographed. Control mice was injected with saline alone $(\mathrm{C})$. The data shown are representative of five different slides. 

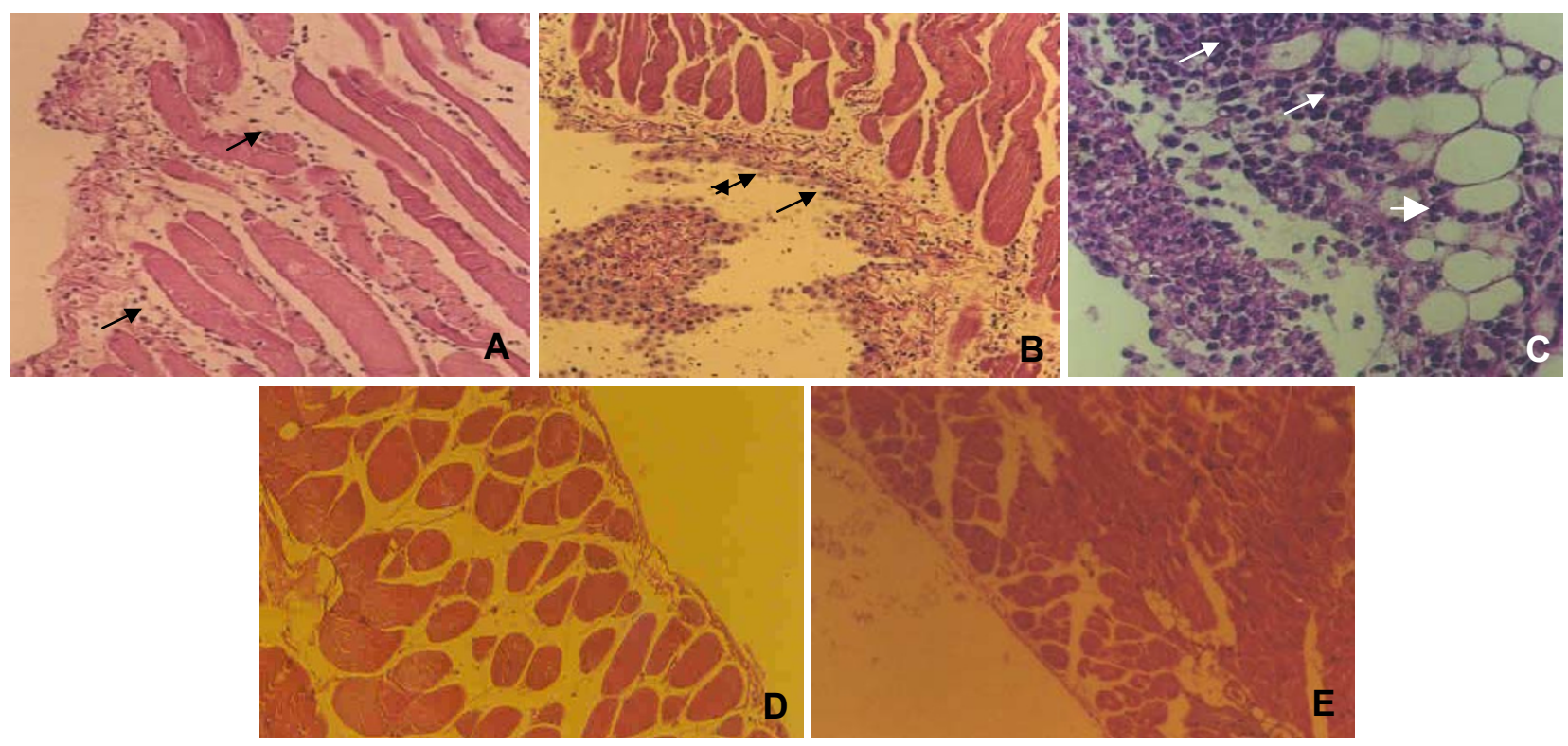

Figure 3. H\&E staining of peritoneal membrane in C57BL/6 mice. Mice were admisistered i.p. with $3 \mathrm{mg} / \mathrm{kg}$ of $\mathrm{rGITR}$ or $50 \mathrm{mg} / \mathrm{kg}$ of LPS in saline. After $24 \mathrm{~h}(\mathrm{~A})$ or $15 \mathrm{~h}$ of GITR injection (B-C) or $15 \mathrm{~h}$ of LPS injection (D), peritoneal membrane was obtained, stored in $10 \%$ formalin, stained by hematoxylin and eosin, and photographed. Infiltrated neutrophil (A), mesothelial cell growth (B), lymphocytes and neutrophil infiltration $(\mathrm{C})$ were indicated by arrows. Control mice was injected with saline alone and peritoneal membrane was obtained after $15 \mathrm{~h}$ of injection (E). The data shown are representative of five different slides.

nates. Specific activity of myeloperoxidase increased about 4 -fold after $8 \mathrm{~h}$ of sGITR stimulation, remained up to $24 \mathrm{~h}$ (Data not shown), supporting the above histochemical data as shown in Figure 3A. Mesothelial cell growth was observed at $15 \mathrm{~h}$ (Figure 3B). At $15 \mathrm{~h}$ lymphocyte and neutrophil infiltration were also observed in omental fat tissue while inflammation is under process (Figure $3 \mathrm{C}$ ).

We wanted to verify that the observed effects of sGITR in mice were genuine and not due to some nonspecific activation or endotoxin contamination associated with the GITR protein preparation. Effects of GITR protein isolated from different sources were tested; GITR proteins produced by the Baculovirus expression system as a fusion protein with polyhistidine tag and those by HEK 293 cells as a fusion protein with Fc. GITR-Fc was injected in mice intraperitoneally at a dose of $0.3 \mathrm{mg} / \mathrm{kg}$ due to a limited supply. It also induced a mild inflammation in peritoneal membrane and increased proportion of red pulp in spleen after $15 \mathrm{~h}$. In addition, infiltration of mononuclear cells such as lymphocyte, macrophage, and plasma cells was observed in omental fat tissue with a less extent (Data not shown). Similar pattern was observed with the GITR protein expressed from Baculovirus (Data not shown).

To exclude the possibility that inflammation is due to LPS-like contaminant from E. coli-produced GITR, the effects of LPS were observed. No significant change in the spleen (Figure $2 \mathrm{~B}$ and $2 \mathrm{C}$ ) and in peritoneal membrane was observed in mice treated with $50 \mathrm{mg} / \mathrm{kg}$ of LPS or PBS alone, respectively (Figure 3D and 3E).

Generally TNFR family express its activity via receptor-ligand interaction. Since the change induced by SGITR was significant in the spleen and peritoneal membrane, the expressions of GITR and GITR ligand on the surface of spleen cells and cells from peritoneal fluid were examined by FACS analysis. GITR ligand and GITR were expressed constitutively on the surface of spleen cells (Figure 4A and 4B). Induction with LPS or PMA did not increase the expression levels of them (Data not shown). Large size cells from peritoneal fluid also expressed GITRL and GITR with much lower level than spleen cells did (Figure $4 \mathrm{C}$ and $4 \mathrm{D}$ ).

\section{Discussion}

GITR was identified as a member of TNFR, and induced after dexamethasone stimulation. Although its function is not clear, GITR appears to protect T cells from apoptosis induced by anti-CD3 mAb. We generated extracellular domain of rGITR to test the effect of the SGITR in mice. When mice were injected with SGITR, spleen was enlarged and the ratio of red and white pulp in the spleen was increased. The actual areas of red and white pulp were not measured, but the area of red pulp appeared to be 

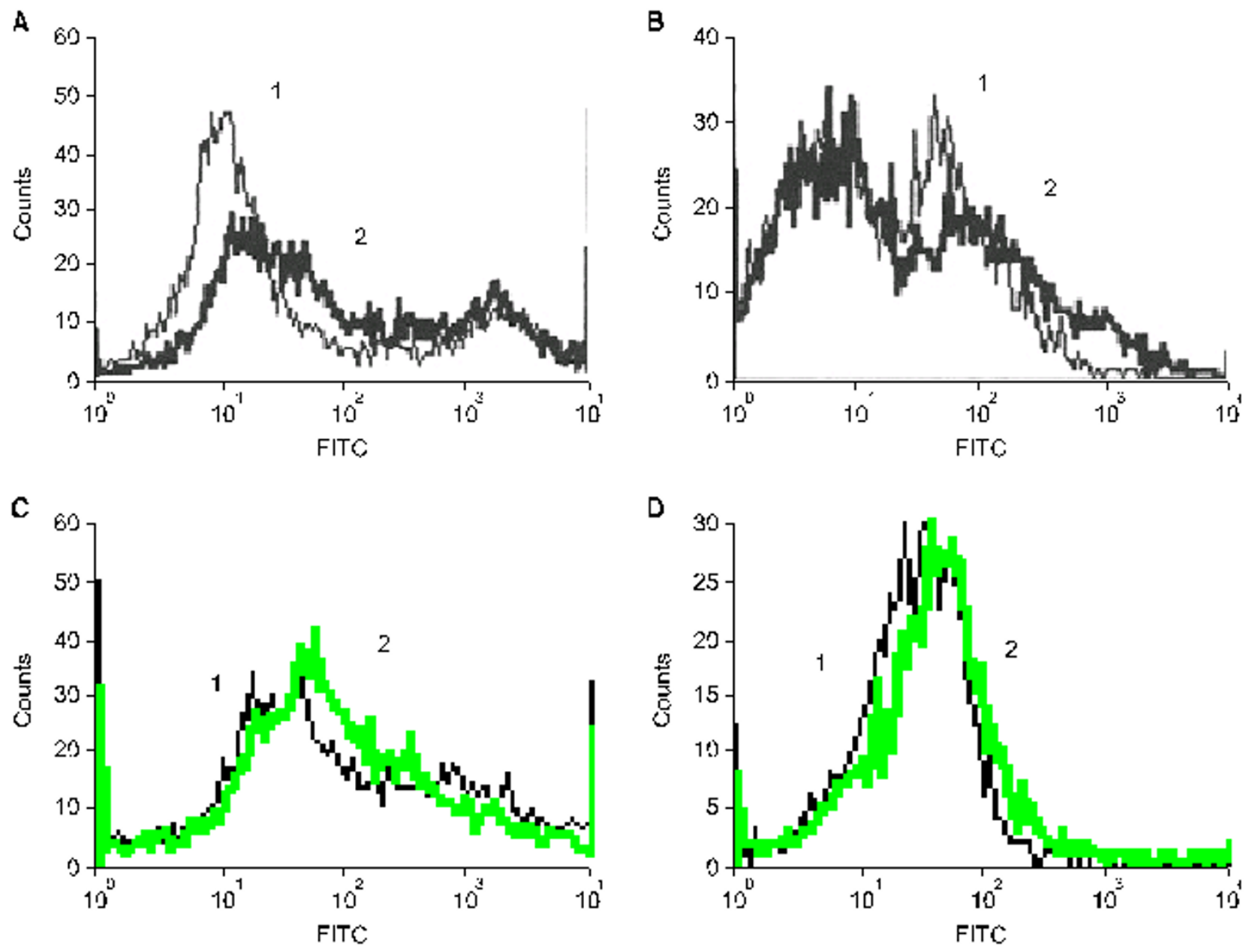

Figure 4. FACS scan of GITR ligand and GITR. For detection of GITR ligand (A), spleen cells were incubated with rGITR, monoclonal anti-histidine $A b$, and FITC-conjugated goat anti mouse IgG (peak 2) each for $30 \mathrm{~min}$ on ice for GITR ligand detection. Cells were washed three times after each incubation. PBS instead of rGITR was used as an isotype control (peak 1). For GITR (B), spleen cells were incubated with polyclonal rabbit anti-GITR $\mathrm{Ab}$ for $30 \mathrm{~min}$ on ice, washed three times, and finally incubated with FITC-conjugated goat anti rabbit lgG (peak 2). Cells treated with preimmune serum were used as an isotype control (peak 1). Cells from peritoneal fluids were prepared by washing peritoneum with PBS (C; GITR ligand, D; GITR).

enlarged due to spleen enlargement. Edema and neutrophil infiltration also observed in the peritoneal membranes. Percentage of peripheral blood neutrophils and monocytes were increased. Infiltrating neutrophils generate oxygen derivatives, serine proteases and zinc matrix metalloproteases (MMPs) to promote tissue injury related to inflammatory processes. Neutrophil infiltration into muscles and fat tissues were followed by muscle destruction. Previously, expressions of MMP-2 and MMP-9 were observed in peritoneal membrane homogenates after GITR injection (Lee et al., 2003). MMP-9 expression appeared to be related to the inflammatory response, whereas MMP-2 activation to be concomitant with the regeneration of new myofibers (Kherif et al., 1999). Growth of mesothelial cells was also observed in peritoneal membrane.

Since SGITR was produced from $E$. coli system, we need to verify the observed effect was from genuine GITR protein, not from contaminated endototoxin of sGITR protein preparation. The GITR preparation contained $<0.4 \mathrm{EU} / \mathrm{mg}$ of endotoxin as determined by E-Toxate assay and the level was quite low. However, we also tried other sources of sGITR protein from Baculovirus system and HEK 293 cells. They also showed similar effect in mice, although it was mild, suggesting that the observed effect was due to SGITR protein. Although sGITR produced inflammatory reaction similar to LPS, there are some differences. For example, when $50 \mathrm{mg} / \mathrm{kg}$ of LPS was injected, no significant changes were found up to $15 \mathrm{~h}$. However, significant level of damage 
was observed in skeletal muscles after $2 \mathrm{~d}$ and inflammation in peritoneum was after $5 \mathrm{~d}$ of LPS treatment (unpublished results).

Soluble forms of TNFR family protein have been demonstrated for TNF receptor family, CD27, CD30, and CD137 (Mohler et al., 1993). These soluble receptor forms are generated in vivo by proteolytic cleavage or alternative splicing. In case of CD27 or CD137 which shared intracellular domains with GITR, their soluble forms were found in inflammatory states with a positive correlation. Soluble CD137 was demonstrated to be released by activated lymphocytes and be detectible in sera of patients of rheumatoid arthritis (Mitchel et al., 1998). Soluble CD27 has been also demonstrated for stimulation of $T$ lymphocytes in patients of multiple sclerosis (Hintsen et al., 1999) and systemic lupus erythematosus (Font et al., 1996).

In this study, presence of GITR and GITR ligand in the surface of mouse spleen cells and cells from peritoneal fluids were demonstrated by FACS. AIthough the expression levels were low, they were constitutively expressed. Taken together, these data indicate that injected sGITR induced inflammatory events in peritoneum and changes of spleen in vivo. It is possible that soluble receptor bind to the membrane bound ligand and gives a retrosignal, an inflammatory reaction. Or soluble GITR acts as an antagonist and blocks a tonic anti-inflammatory effect of GITR ligand. The answer could be waited until GITR ligand has been identified.

Our studies suggest that SGITR participates in the inflammatory process observed in peritoneal membranes. Inflammation is the body's reaction to invasion by infection or physical damage. It represents the consequence of capillary dilation with edema and the emigration of leukocytes. Once the initiating event has occurred, the prolongation of the inflammatory process resulted in a variety of immune responses such as activation of $T$ cell and cytokine expression. In our studies sGITR triggered inflammatory processes in peritoneal membranes and neighboring fat tissues. GITR also change size and structure of the spleen with high ratio of red pulp to white pulp, although it is not clear what indicates those changes in the spleen. It remains to be determined whether the effect of GITR is mediated by GITR ligand upon binding to GITR.

\section{Acknowledgement}

This work was supported by SRC fund to IRC, University of Ulsan from KOSEF and Ministry of Korea Sciences and Technology.

\section{References}

Akiba $H$, Nakano $H$, Nishinaka $S$, Shindo M, Kobata T, Atsuta M, Morimoto C, Ware CF, Malinin NI, Wallach D, Yagita $\mathrm{H}$, Okumura K. CD27, a member of the tumor necrosis factor receptor superfamily, activates NF-kB and stress-activated protein kinase/c-Jun $\mathrm{N}$-terminal kinase via TRAF2, TRAF5, and NF- $\mathrm{kB}$-inducing kinase. J Biol Chem 1998;273:13353-8

Arch $\mathrm{RH}$, Thompson CV. 4-1BB and $0 \times 40$ are members of a tumor necrosis factor (TNF)-nerve growth factor receptor subfamily that bind TNF receptor-associated factors and activate nuclear factor $\kappa B$. Mol Cell Biol 1998;18:558-65

Cannons JL, Choi Y, Watts TH. Role of TNF receptorassociated factor 2 and p38 mitogen-activated protein kinase activation during 4-1BB-dependent immune response. J Immunol 2000;165:6193-204

Font J, Pallares L, Martorell J, Martinez E, Gaya A, Vives $\mathrm{J}$, Ingelmo M. Elevated soluble CD27 levels in serum of patients with systemic lupus erythematosus. Clin Immunol Immunopathol 1996;81:239-43

Goldstein RA, Bowen DL, Fauci AS. Adrenal corticosteroids. In: Gallin JL, Goldstein IM, Snyderman R. (eds) Inflammation: basic principles and clinical correlates, vol. 55, 2nd Ed, 1992;1061-92, Raven, New York

Heinisch IV, Daigle I, Knopfli, B, Simon HU. CD137 activation abrogates granulocyte-macrophage colony-stimulating factormediated anti-apoptosis in neutrophils. Eur J Immunol 2000; 30:3441-6

Hintzen RQ, Paty D, Oger J. Cerebrospinal fluid concentrations of soluble CD27 in HTLV-1 associated myelopathy and multiple sclerosis. J Neurol Neurosurg Psychiatry 1999; 66:791-3

Jacquot S, Kobata T, Iwata S, Morimoto C, Schlossman SF. CD154/CD40 and CD70/CD27 interactions have different and sequential functions in $\mathrm{T}$ cell-dependent $\mathrm{B}$ cell responses: enhancement of plasma cell differentiation by CD27 signaling. J Immunol 1997;159:2652-7

Jang IK, Lee ZH, Kim YJ, Kim SH, Kwon BS. Human 4-1BB (CD137) signals are mediated by TRAF2 and activate nuclear factor- $\mathrm{KB}$. Biochem Biophys Res Commun 1998; 242:613-20

Kwon B, Yu KY, Ni J, Yu GL, Jang IK, Kim YJ, Xinf L, Liu D, Wang S-X, Kwon BS. Identification of a novel activation-inducible protein of the tumor necrosis factor receptor superfamily and its ligand. J Biol Chem 1999;274: 6056-61.

Kwon BS, Weismann SM. cDNA Sequences of two inducible T-cell genes. Proc Natl Acad Sci USA 1989;86:1963-7

Lee HS, Shin HH, Kwon BS, Choi HS. Soluble GITR increased MMP-9 activity in murine macrophage. J Cell Biochem 2003;88:1048-56

Michel J, Langstein J, Hofstadter F, Schwarz H. A soluble form of CD137, a member of the TNF receptor family is released by activated lymphocytes and is detectible in sera of patients of rheumatoid arthritis. Eur J Immunol 1998; 28:290-5 
Mohler KM, Torrance DS, Smith CA, Goodwin RG, Stremier $K E$, Fung VP, Madani $H$, Widmer MB. Soluble tumor necrosis factor TNF receptors are effective therapeutic agents in lethal endotoxemia and function simultaneously as both TNF carriers and TNF antagonists. J Immunol 1993;151: 1548-61

Nocentini G, Giunchi L, Ronchetti S, Krausz LT, Bartoli A, Moraca R, Migliorati G, Riccardi C. A new family of the tumor necrosis factor/nerve growth factor receptor family inhibits $T$ cell receptor-induced apoptosis. Proc Natl Acad Sci USA 1997;94:6216-21
Shin $\mathrm{HH}$, Kwon BS, Choi HS. Recombinant GITR induced COX-2 activity in murine macrophage Raw 264.7 cells. Cytokine 2002;19:187-92

Shin $\mathrm{HH}$, Lee MH, Kim SK, Lee YH, Kwon BS, Choi HS. Recombinant GITR induces NOS in murine mmacrophage. FEBS Lett 2002;514:275-80

Takeda K, Oshima H, Hayakawa $Y$, Akiba H, Atsuta M, Kobata T, Kobayashi K, Ito M, Yagita H, Okumura K. CD27mediated activation of murine NK cells. J Immunol 2000; $164: 1741-5$ 